Cahiers « Mondes anciens »

ANCIENS

Histoire et anthropologie des mondes anciens

5 | 2014

Maudire et mal dire : paroles menaçantes en Grèce ancienne

\title{
Parrhèsia socratique et parrhèsia cynique : le cas de l'injure
}

\section{Suzanne Husson}

\section{OpenEdition}

\section{Journals}

Édition électronique

URL : https://journals.openedition.org/mondesanciens/1256

DOI : $10.4000 /$ mondesanciens. 1256

ISSN : 2107-0199

\section{Éditeur}

UMR 8210 Anthropologie et Histoire des Mondes Antiques

\section{Référence électronique}

Suzanne Husson, «Parrhèsia socratique et parrhèsia cynique : le cas de l'injure », Cahiers « Mondes anciens » [En ligne], 5 | 2014, mis en ligne le 14 février 2014, consulté le 21 septembre 2021. URL : http://journals.openedition.org/mondesanciens/1256; DOI : https://doi.org/10.4000/mondesanciens. 1256

Ce document a été généré automatiquement le 21 septembre 2021.

\section{(c) (i) $\odot$}

Les Cahiers «Mondes Anciens » sont mis à disposition selon les termes de la licence Creative Commons Attribution - Pas d'Utilisation Commerciale - Pas de Modification 4.0 International. 


\title{
Parrhèsia socratique et parrhèsia cynique : le cas de l'injure
}

\author{
Suzanne Husson
}

1 Dans les Lois, Platon est sans appel : l'injure doit être bannie d'entre les citoyens et ceci quel que soit le sujet de dispute :

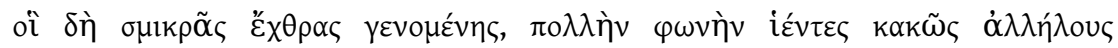

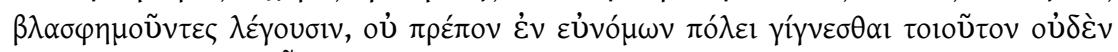

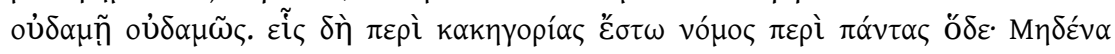

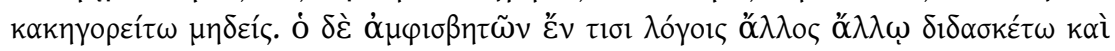

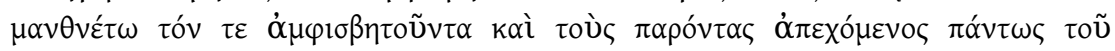

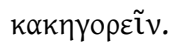

Ce sont eux [c.-à-d. les hommes violents] qui, pour la moindre offense, jettent les hauts cris, se disent les uns aux autres des injures salissantes, et c'est un spectacle qui n'est pas du tout, d'aucune façon et nulle part, convenable dans une cite bien policée. Nous porterons donc une seule loi pour interdire à tous les hommes l'injure. La voici. Que nul n'injurie qui que ce soit. En toute contestation, le débat doit se faire en écoutant les raisons d'autrui et exposant les siennes, tant aux adversaires qu'aux personnes présentes, sans y mêler aucune injure.

(Lois, XI, 934d7-e6, trad. A. Diès)

Or, il est certain que Platon proclame également que le philosophe doit s'exprimer avec franchise. Certes, toute forme de parrhèsia n'est pas légitime. Celle-ci, en effet, ne peut s'exercer légitimement pour Platon que dans les situations de hiérarchie éthique, c'est-àdire celles où le libre locuteur est doué d'une autorité morale incontestable. Ainsi, Lachès, malgré son grand âge, autorise Socrate à user de parrhèsia envers lui, uniquement parce que ses actes de bravoure passés le rendent digne de la liberté de parole ${ }^{1}$. Cela signifie donc que dans une situation normale, les plus jeunes, censés être moins sages, ne sont pas autorisés à formuler ouvertement leur pensée lorsqu'elle met directement en cause le statut de leur interlocuteur. A contrario, l'expérience de l'ivresse provoque une hardiesse qui conduit à se prêter une sagesse et une supériorité morale illusoires et à ignorer ainsi les limites de la parrhèsia ${ }^{2}$. La liberté de parole n'est donc justifiée que venant d'un 
individu moralement supérieur s'adressant à un sujet moins avancé, ou bien alors dans une situation d'égalité morale et de confiance amicale³.

Ces règles de la parrhèsia se retrouvent également au niveau politique. En effet, si c'est bien la liberté de parole qui caractérise la démocratie ${ }^{4}$, celle-ci ne peut être que rejetée par Platon dans ce contexte, puisque le propre de ce régime est de pratiquer un égalitarisme visant à nier toute hiérarchie morale. Dans ces conditions la liberté de parole devient une licence sans frein, comparable à celle de l'ivresse. À l'opposé, la tyrannie, le plus corrompu des régimes, exclut totalement la parrhèsia, puisque le tyran, contrairement au bon roi, en arrive à ne plus l'accepter dans le cercle de ses proches encore doués de courage ${ }^{5}$. La bonne politique implique donc une forme de parrhèsia, par laquelle les gouvernants ne refusent pas d'éduquer moralement le peuple en lui révélant ses défauts ${ }^{6}$, alors que la liberté de parole des gouvernés n'est accordée que selon certaines règles?

4 Mais si dans les situations d'interlocution entre individus, la parrhèsia, lorsqu'elle est légitime, consiste à dire ce que l'on estime être vrai, quel que soit le risque encouru, à faire coïncider ses pensées et ses actes ${ }^{8}$, celle-ci ne requiert-elle pas d'appeler les choses par leur nom et donc de recourir parfois à l'injure? Nous chercherons ici à montrer comment, d'un point de vue philosophique, l'injure, la kaknүopía, apparaît comme l'une des manifestations naturelles du franc-parler. En effet, si Socrate, tel que Platon nous le présente, met en œuvre une stratégie subtile dans le but d'y échapper, les cyniques, refusant d'enfermer la parrhèsia du philosophe dans les conventions de la bienséance, n'hésitèrent pas à y recourir, l'invective directe faisant partie de leur programme de «falsification de la monnaie».

Mais avant de poursuivre, il est peut être nécessaire de préciser ce qu'est l'injure pour Platon.

Si l'injure, en effet, requiert d'être définie c'est avant tout pour des raisons politiques, car elle ne saurait être tolérée dans une cité conforme à la justice. Dès la République, nous

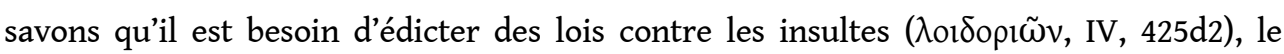
projet n'est cependant mené à bien qu'au livre XI des Lois (934d5-936b2). Cet acte de parole interdit y est désigné par le verbe $\beta \lambda \alpha \sigma \varphi \eta \mu \varepsilon ́ \omega$ (934e1), par le verbe $\lambda{ }_{o 1} \delta \circ \rho \varepsilon ́ \omega$

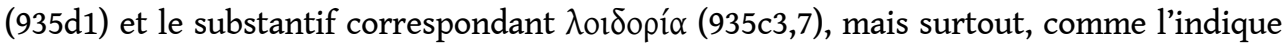

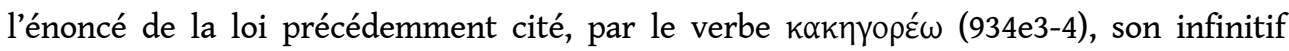

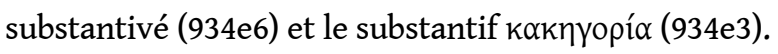

7 Ce qui, pour Platon, caractérise de prime abord l'injure est l'état du locuteur qui se trouve sous le coup de la colère, ou plutôt qui est sous le joug à la fois d'une mauvaise nature

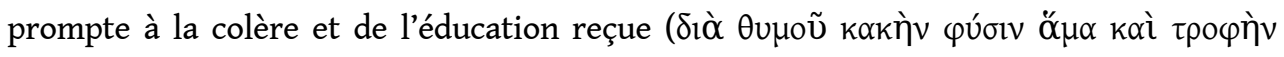

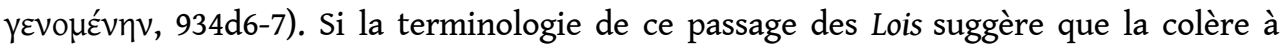

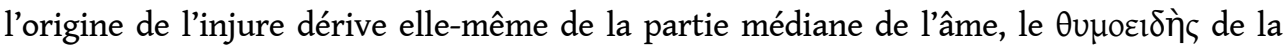
République, qui comme la partie en cause ici, risque, sans éducation, de s'ensauvager ${ }^{9}$, le mythe de l'attelage ailé dans le Phèdre nous montre que cette colère peut aussi provenir de la partie inférieure de l'âme, la partie désirante. Le cheval noir, en effet, "ami de

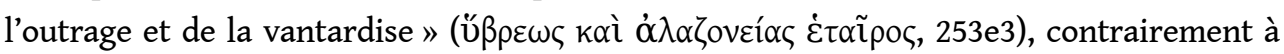
son compagnon d'attelage plus docile, qui représente la partie courageuse, se cabre dès que le cocher, la partie rationnelle, tire les rênes en arrière et « à peine a t-il repris son

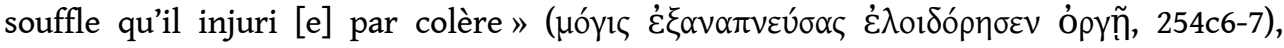

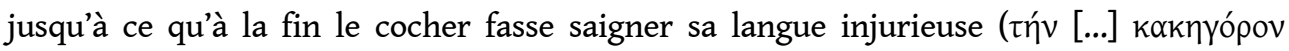


$\gamma \lambda \hat{\omega} \tau \tau \alpha \nu, 254 \mathrm{e} 3)$. La violence propre aux désirs inférieurs, ceux qui dérivent directement du corps, peut donc aussi être à la source de l'injure, sans doute est-ce pour cela qu'elle est plus particulièrement prêtée aux femmes ${ }^{10}$, et aux hommes lâches ${ }^{11}$, c'est-à-dire à ceux dont la partie courageuse de l'âme, faute d'aptitudes naturelles ou d'éducation, est déficiente. L'injure est dans les deux cas une forme de violence déréglée, où des mots

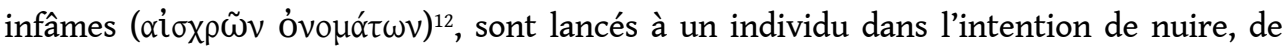
sorte qu'elle engendre elle-même la violence (935a1-7). C'est ainsi que se justifie son interdiction dans la cité, sauf dans les joutes purement ludiques entre poètes (935c7-936b2). Mais si tel est le cas, ne pourrait-on pas concevoir que l'injure puisse également prendre part au processus éducatif, à partir du moment où elle est mue par un noble dessein? Isocrate ne dit-il pas dans le Panégyrique qu'«il est impossible de détourner les fautes ou de pousser à désirer des actes différents si l'on ne blâme pas

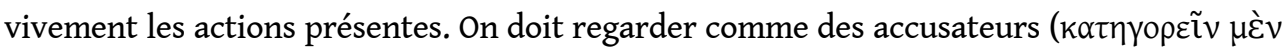
$\dot{\eta} ү \varepsilon \tilde{\imath} \sigma \theta \alpha \mathrm{l})$ ceux qui parlent ainsi pour nuire; mais comme des conseillers ceux qui

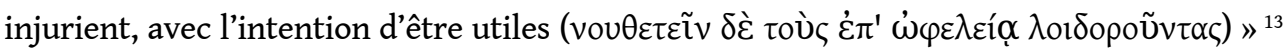
?

Il semble bien que cela ne puisse être le cas pour Platon, comme le portrait qu'il nous donne de Socrate en témoigne. Face à l'insulte, ce dernier pratique en permanence l'art de l'esquive.

9 En effet, si la maïeutique consiste à faire en sorte que les partenaires dialectiques de Socrate comprennent que leurs opinions spontanées sont erronées et sachent qu'ils ne savent pas, il n'est pas étonnant que cette entreprise suscite leur agressivité et que, malgré leur bonne éducation, les insultes parfois affleurent. Par exemple, Thrasymaque, au livre I de la République (343 a), est directement injurieux, et traite Socrate de morveux :

La discussion en était là, et il était devenu évident à tous les assistants qu'elle avait abouti à une définition du juste exactement contraire, quand Thrasymaque, au lieu de répondre, s'écria :

- Dis-moi, Socrate, as-tu une nourrice?

- Quoi ? répliquai-je, ne vaudrait-il pas mieux répondre que de faire de pareilles questions?

- C'est que, dit-il, elle te laisse ainsi morveux, au lieu de te moucher.

(République, 343 a1-8, trad. E. Chambry)

Dans le Gorgias l'atmosphère est à peine plus feutrée, si reproches et objections fusent, ceux-ci en restent la plupart du temps au niveau des considérations générales sur la rhétorique et son caractère illusoire, de telle sorte que l'injure, qui exige qu'un défaut honteux soit attribué nommément à un individu identifié ${ }^{14}$, n'est pas formulée. Néanmoins, au fur et à mesure de la discussion, les patiences s'épuisent. Par exemple dans cet échange entre Polos et Socrate, ce dernier vient de soutenir que les tyrans et les orateurs ne font pas ce qu'ils veulent, même s'ils font ce qui leur plaît, ce qui fait dire à Polos :

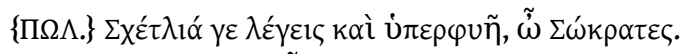

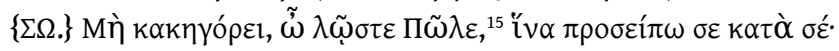

Polos - Ce que tu dis est effroyable et monstrueux, Socrate !

Socrate - Ne profère pas d'injures, noble Polos, pour parler comme toi !

(Gorgias, 467 b10-c1)

11 Socrate est en train de signifier à Polos que les mots qu'il emploie peuvent être injurieux. Mais c'est avec Calliclès que les propos deviennent plus agressifs. Sans l'insulter directement, là non plus, celui-ci lui conseille cependant, en toute amitié, d'abandonner 
la pratique de la philosophie qui, à son âge, est ridicule, « indigne d'un homme et digne de

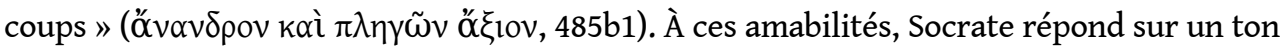
enthousiaste qu'il a enfin trouvé le maître qu'il cherchait :

En effet, je suis convaincu que si l'on doit contrôler une âme et la mettre à l'épreuve pour voir si elle vit bien ou mal, il faut avoir trois qualités; or, toi, tu les as toutes les trois. Il s'agit de la connaissance, de la bienveillance et de la franchise (

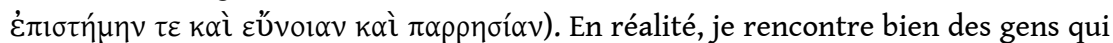
sont incapables de me mettre à l'épreuve, faute d'avoir la connaissance pour cela, alors que toi, tu as cette connaissance. Et d'autres, qui sont savants, mais qui ne veulent pas me dire la vérité, parce qu'ils n'ont pas le moindre intérêt pour moi, tandis que toi, tu t'intéresses à moi. Regarde, les deux étrangers avec qui j'ai parlé, tes amis, Gorgias et Polos ; en effet, ce sont des hommes savants et ils ont de l'amitié pour moi, mais ils n'ont pas assez de franchise, ils éprouvent trop de gêne - en tout

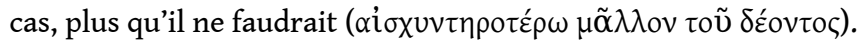

(Gorgias, $487 \mathrm{a}-\mathrm{b}$, trad. M. Canto, très légèrement modifiée)

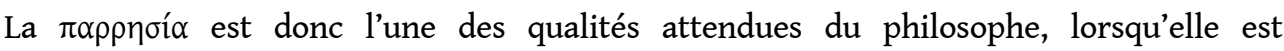
accompagnée de connaissance et bienveillance, et celle-ci implique une certaine levée de la retenue que la société impose ordinairement au discours. Le philosophe ne doit être mû que par la retenue morale, la seule pertinente, et ne doit suivre aucune autre forme de pudeur intéressée ou conventionnelle ${ }^{16}$, de telle sorte que son discours apparaîtra parfois comme grossier (cf. Gorgias, 462e6). Socrate ne reproche donc pas explicitement à Calliclès de l'avoir insulté, car ses paroles sont censées être motivées par l'zúvolo et non par la colère. C'est donc bien l'intention qui fait l'insulte. Le reproche direct, qui serait ordinairement perçu comme une offense insupportable, devient légitime dans un contexte éducatif. Mais encore faut-il que celui-ci existe. Or, Socrate, en réitérant sa profession d'ignorance, le fait immédiatement exister, puisqu'il se met dans la position de l'élève prêt à recevoir sagement des leçons, ce qui désamorce toute situation potentiellement conflictuelle.

Mais n'en fait-il pas trop? C'est en tout cas ce que pense son interlocuteur un peu plus loin :

Socrate - Et puis, homme étonnant, sois plus doux quand tu commences à instruire ton élève, sinon je vais cesser de venir prendre des leçons chez toi.

Calliclès - Tu fais semblant (Ei $\rho \omega v \varepsilon u ́ n)$, Socrate !

Socrate - Je le jure par Zéthos ${ }^{17}$, dont toi-même, Calliclès, tu t'es servi à plusieurs

reprises quand tu faisais semblant ( $\varepsilon i \rho \omega v \varepsilon u ́ o v) . »$

(Gorgias, 489 d7-e3, trad. M. Canto, très légèrement modifiée.)

La réponse de Socrate est subtile, car elle peut être comprise à la fois comme une protestation de sincérité et un aveu de duplicité. Quoi qu'il en soit, Socrate fait savoir à Calliclès que lui, non plus, n'est pas dupe de ses protestations d'amitié. L'ironie est donc utilisée des deux côtés pour désamorcer le caractère injurieux du propos. Si elle est une pure hypocrisie de politesse chez Calliclès, elle est inséparable, chez Socrate, de sa profession d'ignorance ${ }^{18}$.

Mais s'il est certain que, contrairement à ce que feint de croire Socrate, Calliclès ne faisait preuve ni de connaissance, ni de bienveillance, Socrate, par son ironie, ne néglige-t-il pas la franchise ? Pourquoi ne prend-il pas le risque d'énoncer directement les faiblesses de ses interlocuteurs, et de dire à Calliclès ce que le lecteur sait que Socrate pense vraiment, à savoir qu'il est un imbécile prétentieux et malintentionné ${ }^{19}$ ? S'il agissait ainsi, son interlocuteur interromprait immédiatement le débat, or, chez Socrate, la parrhèsia est subordonnée à l'enjeu dialectique. Dire de but en blanc à un individu qu'il est ignorant ne 
le fera pas avancer, il faut donc, avant toute chose, privilégier la continuité de sa participation à l'enquête et à sa propre réfutation. Voilà pourquoi, lorsque l'injure menace, il est urgent de l'esquiver, afin de mener le logos à son terme. Mais lorsqu'une voie de réfutation de la doxa de l'interlocuteur, considérée en elle-même, se présente, il faut la prendre sans honte et sans peur. Ainsi, si, après avoir subi la réfutation, les partenaires peuvent percevoir la démarche de Socrate comme insultante - puisqu'elle les met face à leurs propres contradictions, remet publiquement en question les compétences qui fondent leur statut social et leur fait perdre la face - dans le cours de l'entretien luimême, aucun propos insultant ne sort explicitement de la bouche du philosophe, qui ne quitte pas la position de l'élève désireux d'apprendre, ce qui le rend insaisissable.

La parrhèsia socratique évite donc l'injure pour pouvoir poursuivre jusqu'au bout le dialogue. Mais que devient-elle dans un contexte où la démarche dialectique semble exclue ? Selon le mot de Diogène Laërce (VI, 103), les cyniques délaissèrent la partie dialectique et physique de la philosophie pour ne conserver que l'éthique ; ils reprirent d'autre part, avec panache, le flambeau de la parrhèsia, sans que l'impératif dialectique ne vienne écarter l'injure. Mais, comment celle-ci pouvait-elle être justifiée ?

Tout d'abord l'appellation de l'école semble être, elle-même, issue d'une insulte, comme en témoigne l'anecdote suivante :

Alors que Polyxène le dialecticien s'indignait parce que certains l'interpellaient par le mot « chien », il dit : " Toi aussi appelle-moi “chien”. En effet, Diogène n'est pour moi qu'un surnom ; c'est un chien que je suis, mais de ces chiens de bonne race qui montent la garde sur leurs amis. »

$(\text { Gnomologium Vaticanum 743, n. } 194=\text { SSR VB 149 })^{20}$

La mention précise de Polyxène, laisse penser que nous avons là une chrie remontant sans grande transformation aux premiers disciples de Diogène, et faisant sans doute référence à un épisode historique ${ }^{21}$. Apparemment donc Diogène - qu'on avait appelé « chien ", sans doute du fait de sa pratique des œuvres de Déméter et d'Aphrodite en public - avait assumé ce nom en lui donnant un sens positif : le chien est en effet présenté par Platon au livre II de la République (375d10-e4) comme un animal doué du naturel philosophe parce qu'il sait distinguer les familiers de ceux qui lui sont étrangers ${ }^{22}$. Néanmoins, le chien cynique inverse apparemment le comportement canin habituel car il mord ses amis, mais dans un but éducatif : "Diogène disait: "les autres chiens mordent leurs ennemis, mais moi je mords mes amis afin de les sauver" » (STOB. III 13, 44 =SSR VB 149). L'insulte est parfois aussi comiquement retournée à l'envoyeur :

Alors qu'il mangeait sur la place publique, ceux qui l'entouraient n'arrêtaient pas de dire «chien!». Celui-ci dit: «c'est vous qui êtes les chiens, vous qui vous êtes tenus autour de moi, pendant je mangeais !»

(Diogène Laërce, VI 61 = SSR VB 147)

19 Toutefois, en ce qui concerne le cynisme, il serait bon, d'un point de vue méthodologique, de distinguer, à propos de l'insulte, les témoignages ou fragments d'écrits cyniques qui ont pu survivre, de la légende cynique, à savoir la tradition anecdotique, qui, certes, a en partie un fondement historique, mais qui est surtout le fruit d'une réélaboration rhétorique poursuivie jusqu'à nos jours.

Dans les textes cyniques, nous pouvons mettre en avant le fait que, selon Philodème,

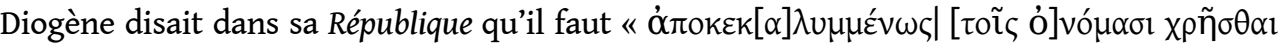

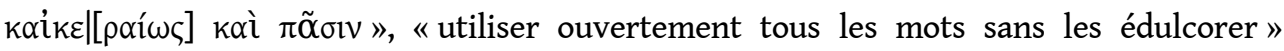
(Philodème, Sur les stoïciens, XVIII 7-8). Cependant, cette disposition ne vise pas 
directement l'injure, mais bien plutôt les mots désignant les parties honteuses et la sexualité. Nous savons, en effet, que Zénon de Kition, inspiré par le cynisme, estimait qu'il n'existe pas de mots obscènes, et qu'il est absurde de pouvoir parler de meurtre et d'adultère - actes condamnables - alors qu'il serait interdit de parler de l'acte sexuel qui, lui, est conforme à la nature ${ }^{23}$. Si donc nous n'avons pas là de justification directe de l'insulte, l'interdit pesant sur certains mots est néanmoins résolument levé.

D'autre part, dans le seul écrit clairement cynique dont il nous reste des extraits conséquents, à savoir Les charlatans démasqués, ou Contre les oracles, d'Oinomaos de Gadara, heureusement - ou providentiellement - conservés par Eusèbe de Césarée, le cynique s'adresse à Apollon, le dieu de la divination, en déconstruisant de façon détaillée la validité de ses oracles ${ }^{24}$. Or, le ton est sans concession, ce qui est d'autant plus

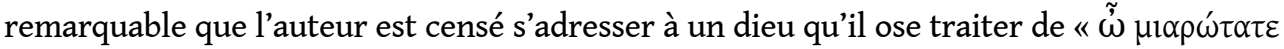

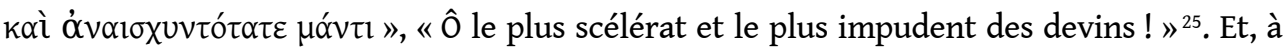
la fin d'un syllogisme établissant que, si tout est déterminé d'avance par le destin, les oracles, qui laissent penser à l'individu qu'il a le choix entre deux options, sont trompeurs, le cynique poursuit :

Si au contraire ce n'est même pas cela, mais qu'il ait dû en advenir ainsi, c'est là le plus impie des propos sophistiques; et s'il devait en advenir ainsi, pourquoi enfin toi, le malheureux Delphien, trônes-tu à débiter tes creux et vains oracles ? Et à quoi nous sers-tu? Pourquoi perdons-nous le sens, nous qui courons à toi de tous les points de la terre ? Toi, qu'est-ce qui te démange?

(Eusèbe, Préparation Évangélique, V, 21, 4 = fr.5. trad. E. des Places, légèrement modifiée)

Oinomaos n'hésite donc pas à traiter le dieu de scélérat, d'impudent, de sophiste. Les reproches injurieux abondent et, suivant le précepte diogénien, sans être édulcorés ${ }^{26}$. Certes nous pourrions penser que le cas de l'invective au dieu est exceptionnel et, peutêtre, trop «facile » pour un esprit fort. Mais, même en supposant qu'Oinomaos ne crût pas en l'existence du dieu qu'il agonissait d'injures, une telle attitude blasphématoire n'était pas sans risque vis à vis des humains.

Si les cyniques, donc, laissèrent parfois un souvenir amer, c'est sans aucun doute parce qu'ils n'hésitaient pas appeler directement les choses par leur nom, sans égard à la susceptibilité de leurs contemporains.

Tournons-nous maintenant vers la légende cynique. Nous y trouvons également une mise en scène de l'injure, mais de façon inattendue celle-ci est relativement rare, sans doute parce que la légende cynique, se devait, du point de vue rhétorique, de provoquer le rire. Or l'insulte à vocation morale, détachée de son contexte d'énonciation, n'est en général pas drôle. Si, sur le moment, il est possible de rire lorsqu'une personne se fait traiter d'« imbécile », le même échange une fois écrit et lu par des lecteurs, à qui les personnes impliquées sont indifférentes, perd toute saveur comique. Ainsi les exemples d'insultes cyniques retenues ou inventées par la tradition sont des jeux de mots, des insinuations et réparties astucieuses, qui se justifient du point de vue littéraire, non pas parce que ce sont des insultes, mais parce qu'elles sont drôles, ou sont censées l'être. Dans le cynisme, l'insulte n'est jamais gratuite, or une insulte à la fois sérieuse et drôle est effectivement rare à trouver. Ainsi, ce sont paradoxalement les exigences du genre comique qui ont limité la présence de l'insulte dans la légende cynique. 
énéral, dans les chries le personnage central ne nomme pas directement les défauts de ses interlocuteurs, mais cherche plutôt à les mettre en lumière, ce qui a un effet bien plus dévastateur.

Il vit un jour une femme qui se prosternait devant les dieux dans une attitude particulièrement indécente. Voulant la débarrasser de sa superstition, à ce que dit Zoïlos de Pergé, il s'approcha d'elle et dit : « Ne crains-tu pas, femme, que si un jour un dieu se tient derrière toi - car tout est rempli de la divinité - ton attitude ne soit indécente?»

(Diogène Laërce, VI 37 = SSR VB 344, trad. M.-O. Goulet-Cazé)

L'insulte qui consisterait à traiter cette fidèle de femme indécente ou superstitieuse, est euphémisée par la formulation d'une question et le raisonnement développé. Dans d'autres contextes, Diogène est plus agressif, mais l'insulte est adoucie par l'à-propos de la répartie :

Il demandait l'aumône à un homme de mauvais caractère, alors qu'il lui disait, « À condition de me convaincre», il dit: « Si je pouvais te convaincre, je te convaincrais d'aller te faire pendre. » (Diogène Laërce, VI 59 = SSR VB 250, trad. M.-O. GouletCazé)

Mais l'une des scènes primitives de la légende diogénienne est, bien sûr, celle-là :

Alors qu'un jour il appelait : «Ohé! Des hommes!» et qu'ils se rassemblaient, il les frappa avec son bâton, en disant, "Ce sont des hommes que j'ai appelés, pas des

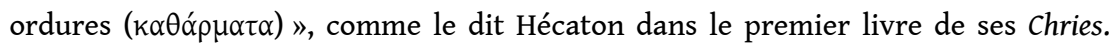
(Diogène Laërce, VI $32=$ SSR VB 278) ${ }^{27}$

À la morsure est joint l'aboiement, au coup porté, l'injure. Pour le cynique, en effet, seuls les sages sont réellement humains ${ }^{28}$, de sorte que l'injure n'est qu'une façon de révéler les hommes à eux-mêmes, de les sortir de leur torpeur et de leurs illusions.

Quoi qu'il en soit, malgré sa relative rareté, l'injure directe est un ingrédient du topos littéraire cynique. Par exemple, celui-ci se caractérise par une absence de pitié pour les hommes «efféminés ». La féminité est, en effet, généralement considérée comme insultante, ce qui montre que la falsification de la monnaie, la contestation cynique des valeurs, a malheureusement des limites :

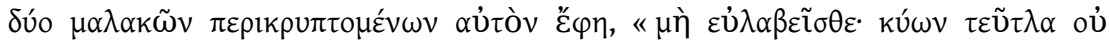

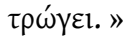

Alors que deux efféminés se cachaient de lui, il dit: «ne vous inquiétez pas, un chien ne dévore pas de bettes. » (DL VI 61 = SSR VB 145)

La mollesse de la bette, par métaphore, en était venue à représenter la "mollesse " morale ${ }^{29}$, et était devenue une insulte. Un autre jeu de mots, plus directement insultant, vise à qualifier, de façon littérale, un jeune homme de prostitué.

Un jour [Diogène] vit un autre [jeune homme] à cheval, de même allure [c'est-à-dire au menton rasé] parfumé et habillé dans un style en accord avec le reste : "Avant ", dit-il, «je cherchais ce que voulait dire "Putain de cheval" (ïrт́́тopvoc), maintenant j'ai trouvé ».

(Athénée, Deipnosophistes, XIII, 565 c = SSR VB 404)

31

Dans le récit suivant, l'insulte est le fait d'un autre cynique, Démonax, qui met à profit l'ambiguïté de óvń $\rho$, « homme » ou « mari », pour traiter sa victime de femme.

Un certain Python qui faisait partie des familles cossues de Macédoine, jeune homme dans la fleur de l'âge, se moquait de lui, lui posait une question sophistique et lui demandait d'énoncer la solution de son syllogisme : «Je sais une seule chose, mon enfant», dit-il, «c'est que tu es pénétrant». Et alors qu'il s'indignait de l'ambiguïté du sarcasme et qu'en même temps il le menaçait : «Je vais, de ce pas, te 
faire voir un homme ( òv $\left.\alpha^{\prime} v \delta \rho \alpha\right)$ ! », et lui en riant lui répondit aussitôt : « C'est

donc que tu en as un?».

(Lucien, Démonax, 15)

À côté de ces insultes à caractère sexuel, on peut trouver également des insultes plus moralisantes, correspondant plus à celles qui pouvaient faire l'objet d'un procès pour kakègoria $^{30}$.

Son mot contre Herminos l'aristotélicien mérite d'être rappelé. En effet, il [Démonax ] savait que ce dernier était un parfait vaurien et qu'il avait accompli des méfaits par milliers, alors qu'il louait Aristote et n'avait que ses dix catégories à la bouche : « Herminos », dit-il, « vraiment, tu es digne des dix catégories ${ }^{31}$ »

(Lucien, Démonax, 15)

Il vit un jour les prêtres gardiens du trésor du temple conduire un individu qui avait dérobé un vase et dit : « les grands voleurs conduisent le petit. »

(Diogène Laërce, VI 45 = SSR VB 462)

L'examen de ces textes montre ainsi que la parrhèsia cynique ne reculait pas devant l'invective directe, et donc l'utilisation de qualifications injurieuses. Les cyniques les plus virulents devaient donc dire leurs "quatre vérités " à leurs contemporains sans trop chercher à les ménager, alors que d'autres, comme Cratès, étaient certainement moins directs. Mais quelle pouvait être la justification philosophique de l'injure? Sans doute faut-il y voir un des aspects du renversement cynique des valeurs véhiculées par la doxa, car, bien évidemment, le cynique s'autorise l'insulte, en premier chef parce qu'il estime être le seul possesseur de la règle morale que nul autre que lui ne respecte. Lorsqu'il

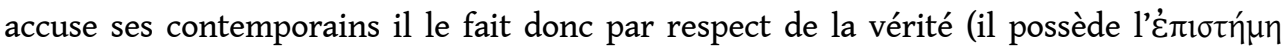
requise par Socrate). Ce sont les lois en vigueur, aussi bien que les usages, qui imposent aux discours une euphémisation respectueuse de la susceptibilité de chacun, les respecter reviendrait à leur reconnaître implicitement une valeur, ainsi qu'à l'honneur et à la réputation publique, pourtant illusoires à la base. En n'évitant pas l'injure, le cynique ne fait que respecter la règle socratique imposant de «ne pas avoir honte plus qu'il ne faudrait ", simplement pour le cynisme les règles qui fondent ordinairement la honte sont contraires à celles que la nature commande. La nature, en effet, est directe, là où la doxa emploie mille circonvolutions. De même qu'elle commande de satisfaire ses besoins physiques de la façon la plus simple, la plus immédiate et la plus directe, il en va de même pour le langage : la vertu et le vice doivent être nommés sans détour. Il n'en va pas de la sincérité de l'individu, qui ici importe peu, mais de la vérité naturelle qui se manifeste à travers lui.

Cependant, peut-on accorder au cynique l'Ev้voı qui l'autoriserait à pousser la franchise jusqu'à l'insulte ? En quoi mordre ses amis peut-il permettre de les sauver? Sans doute, si nous détachions l'injure cynique du mode de vie qui la porte, celle-ci perdrait-elle toute sa valeur et ne serait qu'un acte de misanthropie. C'est bien parce que le cynique commence par s'exposer lui-même à l'injure et montre comment la supporter, qu'il peut l'administrer également à autrui, afin de l'engager à devenir son égal.

Ainsi, si la parrhèsia socratique cherche à éviter l'injure, cela n'implique pas que l'invective directe soit par principe exclue de la franchise de l'amant de la sagesse, bien au contraire. Cette ressource, écartée par les exigences psychologiques de la démarche dialectique, fut, à l'inverse, pleinement assumée par les cyniques, qui ainsi réalisèrent dans toute sa radicalité et sans concession, la nécessité de dire le vrai pour réformer les hommes.

Cahiers « Mondes anciens », 5 | 2014 


\section{BIBLIOGRAPHIE}

Brancacci A. (2000), « Libertà et fato in Enomao di Gadara », dans Brancacci A. dir., La filosofia in età imperiale, Naples, p. 39-67.

Brancacci A. (2001), « La polemica antifatalistica di Enomao di Gadara », dans Brancacci A. dir., Antichi e moderni nella filososofia di età imperiale, Naples, p. 71-110.

Burnet J. et Dodds E.R. (1959), Plato, Gorgias. A revised Text and Commentary, Oxford.

Foucault M. (2009), Le courage de la vérité, Paris.

Gernet L. (1917), Recherches sur le développement de la pensée juridique et morale en Grèce, Paris.

Giannantoni G. (1990), Socratis et socraticorum reliquiae, t. II, Naples.

Glotz G. (1899), « kakègorias dikè », dans Darenberg Ch. et Saglio E. dir., Dictionnaire des antiquités grecques et romaines, Paris.

Hourcade A. (2009), « Les qualités du conseiller : savoir, bienveillance, franc-parler (Platon, Gorgias, 486 e 6-487 d 7) », Dissertatio Revista de filosofia 29, p. 74-75.

Husson S. (2011), La République de Diogène. Une cité en quête de la nature, Paris.

Huston N. (2002), Dire et interdire, Paris.

McKim R. (1988), « Shame and Truth in Plato's Gorgias », dans Griswold Ch. L. éd., Platonic Writings, Platonic Readings, New York, p. 38-39.

Müller R. (2012), « Polyxène », Dictionnaire des philosophes antiques, t. Vb, Paris.

Naber S.A. (1908), « Platonica », Mnemosyne.

Romeyer Dherbey G. (1990), « Zénon appelle les choses par leur nom, la chasteté de la langue d'après les stoïciens ", Mesure 3, p. 47-59.

Vlastos G. (1991), Socrate, Ironie et philosophie morale, Paris.

\section{NOTES}

1. Lachès, 188c4-188a5, cf. Hourcade 2009.

2. Cf. Platon, Phèdre, 240e3-7, Lois, 649b3-5, 671b3-5.

3. Cf. Platon, Lachès, 178a4-179c3.

4. Cf. Platon, République, 555b4-6.

5. Ibid. 5673-6, Cyrus est, comme chez Xénophon, l'exemple du bon roi admettant la liberté de parole (Platon, Lois, 694a2-b7) chez ses subordonnés.

6. Encore faut-il que cette éducation morale tienne compte des limites psychologiques du peuple qui, enfermé dans le cercle de l'opinion, ne pourra jamais accéder à la connaissance. Toute vérité ne peut pas lui être révélée car il serait incapable d'en comprendre tous les enjeux de sorte qu'elle lui serait nocive, le mensonge peut donc se révéler utile (Platon, République 382c6-d4). Ainsi l'existence de hiérarchies morales réelles entre les individus ne peut être acceptée par les gouvernés - non philosophes et ignorant les structures de l'âme humaine - que sous la forme 
d'un mythe fantastique, apte à fixer dans leur âme une vérité morale, sous la forme d'une opinion droite (Ibid. 414b8-415d5).

7. Ainsi, tous les poètes n'auront pas la liberté de parole (Platon, Lois, 829d4-6).

8. Cf. Foucault (2009, p. 14) : «La parrhèsia est donc, en deux mots, le courage de la vérité chez celui qui parle et prend le risque de dire, en dépit de tout, toute la vérité qu'il pense, mais c'est aussi le courage de l'interlocuteur qui accepte de recevoir comme vraie la vérité blessante qu'il entend".

9. Platon, République, 375a11-b10 ; Lois, 934e7-935a7.

10. Cf. Platon, Lois $934 \mathrm{e} 8$; République, $395 \mathrm{~d} 7$.

11. Cf. Platon, République, $395 \mathrm{e} 8$.

12. Platon, Lois, 934e4-935a1, peut-être s'agit-il d'une allusion au catalogue de mots interdits (les

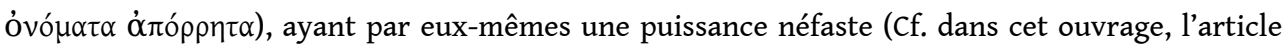
introductif de Vincent Azoulay et Aurélie Damet et l'article de Francis Larran ; voir aussi Gernet 1917, p. 238).

13. Isocrate, Panégyrique, 130, trad. G. Mathieu, légèrement modifiée.

14. En effet, s'efforcer de maintenir le logos au niveau de l'enquête sur un universel est une façon efficace de conjurer l'insulte, qui, elle, exige un énoncé dont le sujet est singulier. Ainsi, démontrer que les prétentions de la rhétorique sont erronées ne peut pas être considéré comme une insulte, même si ce discours est désagréable à entendre, il n'en irait pas de même si Socrate traitait de but en blanc Gorgias et Polos d'idiots ou de menteurs.

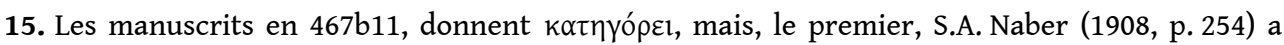
douté de la justesse de cette leçon, tout d'abord parce qu'il s'agirait d'un genre d'accusation

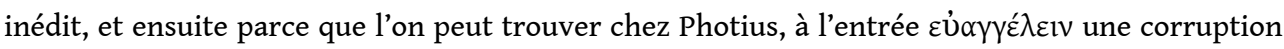

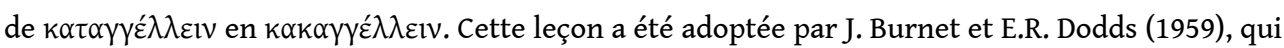
ajoute d'autres occurrences de confusions similaires dans le texte de Platon. Avec $\tilde{\omega} \lambda \tilde{\omega} \sigma \tau \varepsilon \Pi \tilde{\omega} \lambda \varepsilon$ nous avons ici un exemple du style ampoulé de Polos évoqué par Socrate dans le Phèdre (267c) et parodié auparavant dans le Gorgias (448c). Celui-ci se caractérise en particulier par les paronomases, dont nous avons ici un exemple (cf. Naber 1908).

16. Comme le montre A. Hourcade (2009, p. 73-74). R. McKim (1988, p. 38-39) montre que Calliclès estime que toute forme de honte (aidôs) est conventionnelle et donc doit être bannie, alors que pour Socrate la honte, par elle-même, est moralement justifiée.

17. Il s'agit d'un personnage de l'Antiope d'Euripide, dont Calliclès s'est servi pour faire l'apologie de son idéal de vie (cf. Platon, Gorgias, 484e4-485a3, 485e1-486a7).

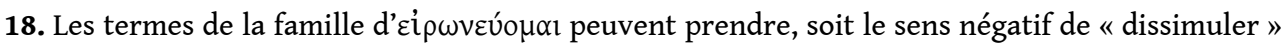
dans l'intention de tromper l'adversaire, soit le sens moderne de s'exprimer de telle sorte qu'on fait entendre ou autre chose, ou le contraire, de ce qui est explicitement énoncé, mais sans intention dissimulatrice. Ici, Calliclès reproche à Socrate de faire semblant d'être son élève, alors que le cours de la réfutation montre au contraire qu'il est le maitre. Autrement dit, Calliclès pense que Socrate n'est pas sincère, et cherche à le tromper sur ses véritables intentions, qui ne sont pas de s'instruire auprès de lui, et Socrate lui retourne le reproche. Cependant la position de Socrate, lorsqu'il fait profession d'ignorance, est plus subtile. G. Vlastos (1991, p. 50-51) l'analyse comme une ironie complexe où « ce qui est dit correspond et en même temps ne correspond pas à ce qui est signifié. Le contenu apparent doit être entendu comme vrai dans un sens et faux dans un autre ", et c'est à l'interlocuteur d'en déterminer le sens : Socrate, dans la mesure où il ne sait rien, d'après sa profession d'ignorance, désire apprendre quelque chose de Calliclès, mais en même temps il n'est pas son élève, puisqu'il ne va rien apprendre de lui. On pourrait dire que, selon l'interprétation de l'interlocuteur, il peut être à la fois ironique, platement sincère ou trompeur. Signalons, que nous divergeons néanmoins de l'interprétation que G. Vlastos donne de ce passage (489 d7-e3 : cf. Vlastos 1991, p. 43-44). 
19. En tout cas en $499 \mathrm{c} 2-4$, Socrate en vient à dire explicitement que Calliclès prend plaisir à le tromper et n'est pas son ami. Il est donc clair que Calliclès ne possède ni la connaissance, ni la bienveillance, quant à la suite du dialogue, elle montre que sa parrhèsia peut également avoir ses faiblesses (McKim 1988, p. 40-43).

20. SSR = Socratis et Socraticorum Reliquiae, G. Giannantoni (ed.), 1990, Naples.

21. Polyxène est un penseur mégarien contemporain de Platon et de Diogène, on lui attribue une version de l'argument du troisième homme (cf. Müller 2012).

22. Cf. Husson 2011, p. 48-53.

23. Cicéron, Les devoirs, I, 35, 128 = SSR VB 515. Cf. Romeyer Dherbey 1990.

24. Certes la distance historique qui sépare cet écrit du II ${ }^{\mathrm{e}} \mathrm{s}$. ap. J.-C. d'un Socrate ou d'un Diogène est importante, mais même si l'ouvrage prend la forme littéraire d'une dénonciation des oracles que l'on peut faire remonter à Hérodote (Histoires, I, 46-81), celle-ci s'inscrit explicitement dans le débat philosophique contemporain portant sur la question de la divination et du destin, les principaux adversaires d'Oenomaus étant les stoïciens (Cf. Brancacci 2000 et Brancacci 2001).

25. Oinomaos chez Eusèbe, Préparation Évangélique, V, 20, 4 (fr. 4), trad. E. des Places.

26. Même si un des passages conservés révèle qu'il pense en fait surtout s'adresser aux charlatans humains qui, en réalité, produisent les oracles (Eusèbe, Préparation Évangélique, VI, 7, 11 = fr. 16).

27. Il existe beaucoup de versions de cette anecdote, recueillies par Giannantoni 1990.

28. Cf. Diogène Laërce, VI, $60=$ SSR VB 273.

29. À moins qu'il ne s'agisse également d'un défaut physique. La même anecdote est répétée en Diogène Laërce, VI, 45 (= SSR VB 145). Pour la constitution d'insultes de façon littérale ou métaphorique, cf. Huston 2002, p. 109-110.

30. Cf. Gernet 1917, p. 234 : «La répression de l'injure verbale dans le droit d'Athènes ne vise que des cas particuliers. Sont punies l'injure aux morts ; l'injure proférée dans un certain nombre de lieux ou d'assemblées (sanctuaires, tribunaux, locaux occupés par les magistrats, théories des fêtes religieuses) - l'emploi d'un certain nombre de termes offensants dont la liste est dressée par la loi ( $\alpha \dot{\pi} \rho \rho \eta \tau \alpha) »$. Ces termes interdits correspondent à des actes interdits par la loi, que l'on a pas le droit de reprocher à un individu sans intenter d'accusation formelle, ou bien des actes permis ou encouragés par la loi (par exemple le fait d'être un boutiquier), qui ne peuvent donc être un motif de reproche. Pour le contenu de cette liste des óđó $\rho \rho \eta \alpha$, voir Glotz 1899.

31. Nous avons ici encore un jeu de mots dû au fait que, dans le titre de l'ouvrage d'Aristote, les " catégories » ont pris le sens de "prédicats » ou "prédications », au-delà du sens originaire du

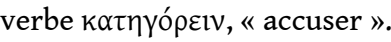

\section{RÉSUMÉS}

L'exercice de la parrhèsia philosophique autorise-t-il le recours à l'injure? Pour Platon, il apparaîtrait à première vue que non, mais l'analyse révèle que l'injure à visée éducative n'est pas rejetée pour des raisons de principe mais parce qu'elle constitue une entrave à la bonne marche de l'entretien dialectique. Dans le cynisme, à l'inverse, l'objectif dialectique étant absent, l'injure devient un moyen admis de manifester la vérité morale. Il $\mathrm{y}$ a, chez Platon, deux cas où la franchise est légitime : lorsqu'un individu moralement supérieur s'adresse à moins avancé que lui, ou lorsque l'on se trouve dans une situation d'égalité amicale. Quant à l'injure, bannie de la cité dans les Lois, elle est constituée de "mots infâmes » lancés sous le coup de la colère, dans 
l'intention de nuire. C'est bien cette colère qui conduit parfois les interlocuteurs de Socrate, et en particulier Calliclès dans le Gorgias, à l'injurier, mais ce dernier désamorce leur agressivité, en attribuant les propos outrageants à la parrhèsia et à la bienveillance éducative de ses interlocuteurs. Les reproches directs, énoncés en public, ne sont donc pas exclus par principe de la démarche éducative, mais Socrate les évite pour pouvoir poursuivre l'entretien jusqu'au bout. Dans le cynisme, l'usage de l'injure à visée éducative est revendiqué, le nom de l'école est luimême issu d'une injure et celle-ci est présente aussi bien dans les textes cyniques que dans la tradition des chries. Il s'agit d'appliquer aux actes de paroles, le refus des règles sociales conventionnelles, non pas en vue d'une spontanéité d'expression sans limite, mais en vue du progrès moral dans le cadre de la parrhèsia philosophique.

May philosophical parrhesia be a good reason to insult our fellow men? For Plato, it would seem, at a first glance, that it is not the case, but further analysis shows that educative insult is not rejected for reasons of principle, but because it seems to be an impediment to dialectical progress. Conversely, in ancient Cynicism, where there is no dialectical aim anymore, insult is a rightful way to show moral truth. For Plato, there are only two cases of rightful frankness : when a morally superior person speaks to someone of a lower level (in an ethical sense) or when the two are equal. Insult is suppressed in Plato's city, as it is drawn up in the Laws, because these "abusive words" are expressed by anger, in order to be harmful. Anger in the cause why interlocutors of Socrates (particularly Callicles in the Gorgias) become insulting, but Socrates defuses it when he interprets their insults as results of parrhesia and educative benevolence. So direct criticism, expressed in public, is not excluded from education on principle, but Socrates avoids it in order to carry on the dialectical work. In Cynicism, educative insult is celebrated, both in cynic literature and in the chreiai, and the name of the school was at the beginning an insult. Rejection of conventional social rules is applied to speech acts, but the aim is not to allow a spontaneous expression of the self without any limitation, but to improve moral progression, as part of the parrhesia.

\section{INDEX}

Mots-clés : Socrate, dialogue, dialectique, cynisme, parrhèsia, Platon, politique, colère, education

Keywords : Socrates, dialectic, cynicism, Plato, politics, anger

\section{AUTEUR}

\section{SUZANNE HUSSON}

Université Paris-Sorbonne, Centre Léon Robin - UMR 8061 